\title{
PREVALENCE, SOCIODEMOGRAPHIC FACTORS AND CLINICAL CRITERIA OF TRICHOMONAS VAGINALIS INFECTION AMONG SYMPTOMATIC WOMEN IN BENI-SUEF GOVERNORATE, EGYPT
} By

\author{
DOAA A. HAMDY ${ }^{1^{*}}$ and HANAN G. HAMDY ${ }^{2}$
}

Department of Medical Parasitology ${ }^{1}$, Faculty of Medicine, Beni-Suef University, and Department of Obstetrics and Gynacology ${ }^{2}, \mathrm{MOH}$ General Hospital, Beni-Suef, Egypt

( ${ }^{*}$ Correspondence: doaahamdypara@gmail.com)

\begin{abstract}
One hundred female patients (age 18-50yrs) were enrolled in this study. Females were nonmenstruating and non-pregnant with no intercourse or douching for the last 2 days, no use of antiprotozoa, antibiotics and steroids for the past 2 weeks and complaining mainly of vaginal discharge, itching or both. Vaginal swabs were obtained from all patients and examined for Trichomonas vaginalis by direct wet mount, Giemsa staining, modified Diamond's culture medium and Latex agglutination test (Kalon). Results: Out of 100 patients, T. vaginalis was found in 8 (8\%) patients by any of the used diagnostic methods. Latex agglutination test detected 8 positive cases, 6 of which were also positive by Diamond's culture, 3 cases were detected by Giemsa staining and only one case was detected by wet mount. The wet mount, Giemsa staining and Kalon latex test had sensitivities of $16.7 \%$, $50 \%$ and $100 \%$ respectively and accuracy rates $95.2 \%, 97.1 \%$ and $98 \%$ respectively as compared to the modified Diamond's culture as the gold standard. Meanwhile all of them had specificities $100 \%$ except for latex agglutination test, it was $97.9 \%$. There was no significant association between potentially probable risk factors and confirmed trichomoniasis detected by Diamond's culture (the gold standard test) except in patients being from urban areas and complaining of either dysuria or dyspareunia or both.
\end{abstract}

Keywords: Trichomonas vaginalis, Wet mount, Diamond's culture, Latex test.

\section{Introduction}

Trichomonas vaginalis is a flagellated anaerobic protozoan parasite that inhabits the urogenital tract of humans (Kaul et al, 2004). Although trichomoniasis is one of the most important sexually transmitted infections (STIs) all over the world that has significant adverse health outcomes, it is the most common curable one (Mabey et al, 2006). The estimated global incidence of T.vaginalis infection was 276.4 million new cases per year (WHO, 2012).

Female infected patients with $T$. vaginalis infection have a wide range of clinical symptoms, ranging from an asymptomatic to an acute or chronic inflammatory disease (Sherrard et al, 2011). This disease plays an important role in causing tubal factor of infertility, ectopic pregnancy and adverse outcomes of pregnancy in infected patients such as premature rupture of membranes, preterm labor and delivery of low-birth weight infants (Cotch et al, 1997; Chesson et al, 2004; Pararas et al, 2006). Trichomoniasis is related to increased risk of HIV-type1 acquisition and transmission (Wang et al, 2001; McClelland et al, 2007). Also, T. vaginalis has been incriminated in vaginitis, urethritis, cervicitis, pelvic inflammatory disease and prostatitis in patients infected with HIV (Moodley et al, 2002). The prostate cancer was associated with male trichomoniasis (Sutcliffe et al, 2006; Stark et al, 2009; Twu et al, 2014).

Control programs for $T$. vaginalis infection had become back behind efforts to control other STIs, that's why T. vaginalis was classified as a neglected parasitic infection (Secor et al, 2014). Many risk factors are associated with $T$. vaginalis infection such as age, race, residence, number of sex partners, marital status, education, intrauterine device (IUD) /condom usage or other used contraceptives, any used drug, presence of vaginal discharge and history of sexually transmitted diseases (Madhivanan et al, 2009; Dahab et al, 2012; Eshete et al, 2013; Miranda et al, 2014; Ton Nu et al, 2015). 
Conventional diagnosis of $T$. vaginalis infection depends on microscopic wet mount examination, in which trophozoite "corkscrew" motility is obvious (Huppert et al, 2007) and Modified Diamond culture which has been used as the gold standard for diagnosis of $T$. vaginalis infection (Harp and Chowdhury, 2011).Both methods suffer from poor sensitivity and rely on collection of fresh viable trichomonads. Other diagnostic used methods include enzyme-linked immuno-sorbent assay (Sibau et al, 1987), staining, immunochromatography and nucleic acid amplification tests (Chalamilla et al, 2006), latex agglutination test (Darani et al, 2010). Ideal diagnosis should depend on high sensitive and specific, available, simple, and inexpensive tests (Patel et al, 2000).

This study aimed to determine prevalence of $T$. vaginalis and risk factors among suspected women attending the Gynecology and Obstetrics Outpatient Clinic at BeniSuef General Hospital using conventional diagnostic methods as wet mount examination, stain, culture and Latex agglutination test. Also, accuracy of direct wet smear, Giemsa stain and Latex agglutination test were evaluated as compared to the gold standard; Diamond's media culture.

\section{Materials and Methods}

Study population: This cross-sectional study was carried out from April 2016 to February 2017on 100 non-menstruating and non-pregnant female patients attending the Gynecology and Obstetrics Outpatient Clinic at Ben-Suef General Hospital, Egypt. The patients were 18 to 50 years old with no intercourse or douching for the last 2 days, no use of antiprotozoal, antibiotics, or steroids for the past 2 weeks and complaining of variable gynecological complaints including mainly vaginal discharge, itching, or both.

Relative sociodemographic and clinical data were collected from all enrolled patients using standardized questionnaire sheet that fulfill the following data about residence, age, marital status, parity, educational level, menstrual and contraceptive history.
Sample collection: Each patient was subjected to PV examination and non-lubricated sterile speculum examination. Three vaginal swabs were collected from the posterior vaginal fornix using a sterile vaginal swab (a cotton wool swab-sticks with wooden shafts) to be examined as follows: 1- The first swab was kept in tube containing $3 \mathrm{ml}$ prewormed sterile Phosphate Buffered Saline (PBS), pH: 7.2, and squeezed into a tube wall to be used, through one hour, for wet mount microscopy and Giemsa staining. 2The second swab was kept in one of the Kalon TV latex tubes provided by the kits containing sterile PBS, $\mathrm{pH} 7.2$, to perform the latex agglutination. 3 . The third swab was immersed immediately into the culture tube containing modified Diamond's medium and squeezed for cultivation. Samples were labeled and rapidly transported to the Medical Parasitology Laboratory for processing.

Wet mount smear: within 10-30 min, one drop of the first swab tube was put into a microscopic clean glass slide then covered by a cover slip and examined microscopically for motile $T$. vaginalis trophozoite under low and high powers.

Giemsa-stained smear: one drop of the vaginal swab-PBS suspension was applied on a microscopic glass slide, air dried, fixed in methanol for 1 minute, stained with $20 \%$ Giemsa for 20 minutes. The slide was rinsed by water to remove excess stain, drainedin a vertical position and left to dry and examined microscopically by X100 to detect trophozoites (Radonjic et al, 2006).

Kalon Latex agglutination test (Kalon Biological Ltd): the latex is mixed on a slide with the elute from the vaginal swab. Presence of $T$. vaginalis antigen in the sample led to agglutination of the sensitized latex. The agglutination of positive samples started to appear after the $2^{\text {nd }}$ minute and established at the $3^{\text {rd }}$ minute. Agglutination of the beads was an indicator for T. vaginalis. The degree of agglutination was recorded following the manufacturer's instructions to distinguish between highly positive, weak positive 
and negative results.

In vitro cultivation of $T$. vaginalis: Directly, the swab was inserted into the culture tubes containing the modified Diamond's medium and after mixing, expressed on the tube side and discarded. The tubes of inoculated medium were incubated vertically at $37^{\circ} \mathrm{C}$ in anaerobic condition. It was examined daily or every other day for the trophozoites. If the parasite was not seen until the $7^{\text {th }}$ day, specimen was considered negative and discarded.

Statistical analysis: Data were tabulated and analyzed using SPSS-23 (IBM, Somers, NY, USA) software. Numerical data were presented as mean $\pm \mathrm{SD}$ while categorical data were expressed as number and percentage. The qualitative variables were studied by chi-square (X2) test. $P$-value was significant at $\leq 0.05$. Receiver operating characteristic curve (ROC) was used to assess the diagnostic performances of the different techniques used in diagnosis.

Ethical approval: All procedures performed in studies involving human participants were in accordance with the ethical standards of the National Research Committee and with the 1964 Helsinki Declaration and its later amendments or comparable ethical standards. All the participants gave informed consent to do vaginal swabbing after explaining the study purpose.

\section{Results}

Out of 100 female patients with symptoms suggestive of vaginal trichomoniasis using the mentioned methods (Tab. 1), positive $T$. vaginalis infection was detected in $8(8 \%)$ of patients by any of the used diagnostic tests. Kalon Latex agglutination test detected trichomoniasis in 8 cases $(100 \%$ of positive cases) of which 6 cases (75\%) were positive by Diamond's culture. While 3 cases and only one case $(37.5 \%$ and $12.5 \%$ respectively) were positive by Giemsa staining and wet mount. These results were highly statistically significant $(P<0.001)$.

Receiver operating characteristic curve (ROC) with analysis of sensitivity and speci- ficity of the used different diagnostic techniques was assessed. A perfect discrimination test has a ROC plot that directed more to the upper left corner $(100 \%$ sensitivity, $100 \%$ specificity). In this study, culture method was used as a gold standard. Females were grouped according to their ages into 4 groups with an age range between 18 \& 50 years and a mean of $32.3( \pm 9.7)$ years. The highest positivity was in age group 31-40 .

No positive cases were reported after the age of 40 years. No statistical significant difference detected in distribution of trichomoniasis in different age groups $(P=0.2)$.

Obtained results clearly showed that $T$. vaginalis infection was more common among women living in urban areas and that was statistically significant $(P=0.006)$. Vaginal discharge, dyspareunia and lower abdominal pain associated with backache were the most frequent symptoms (100\%) among 6 positive detected cases by Diamond's culture method, followed by itching ( $83.3 \%$ ), and then the least frequent symptom was dysuria $(66.7 \%)$.

In this study, $2 / 8(25 \%)$ out of the positive cases detected by Kalon latex test were represented by vaginal discharge only. While there wasn't any positive case found among women complaining of infertility at all. Among all positive cases, a malodorous frothy purulent yellowish green vaginal discharge was detected in 6 cases $(75 \%)$. Two cases were represented by a non-purulent yellowish discharge. Meanwhile yellowish white, bloody and watery vaginal discharge was not related to T.vaginalis infection. Association between the nature of the vaginal discharge and trichomoniasis revealed a highly significant difference $(P<0.001)$.

Of 8 positive cases, 4 cases were using IUD $(50 \%), 2$ cases were using hormonal contraception (25\%) and 2 cases used no contraception $(25 \%)$. This showed insignificant correlation between infection and contraceptives usage. There was insignificant association between sociodemographic risk factors and trichomoniasis by Diamond's 
culture except those living in urban areas $(P=0.006)$ with dysuria $(P=0.003)$, or dyspa- reunia $(P<0.001)$ or both. Details were given in tables $(1,2 \& 3)$ and figures $(1 \& 2)$.

Table 1: Accuracy of direct wet mount, Giemsa staining technique and Kalon Latex agglutination test versus Diamond's culture, gold standard test for diagnosis of vaginal trichomoniasis.

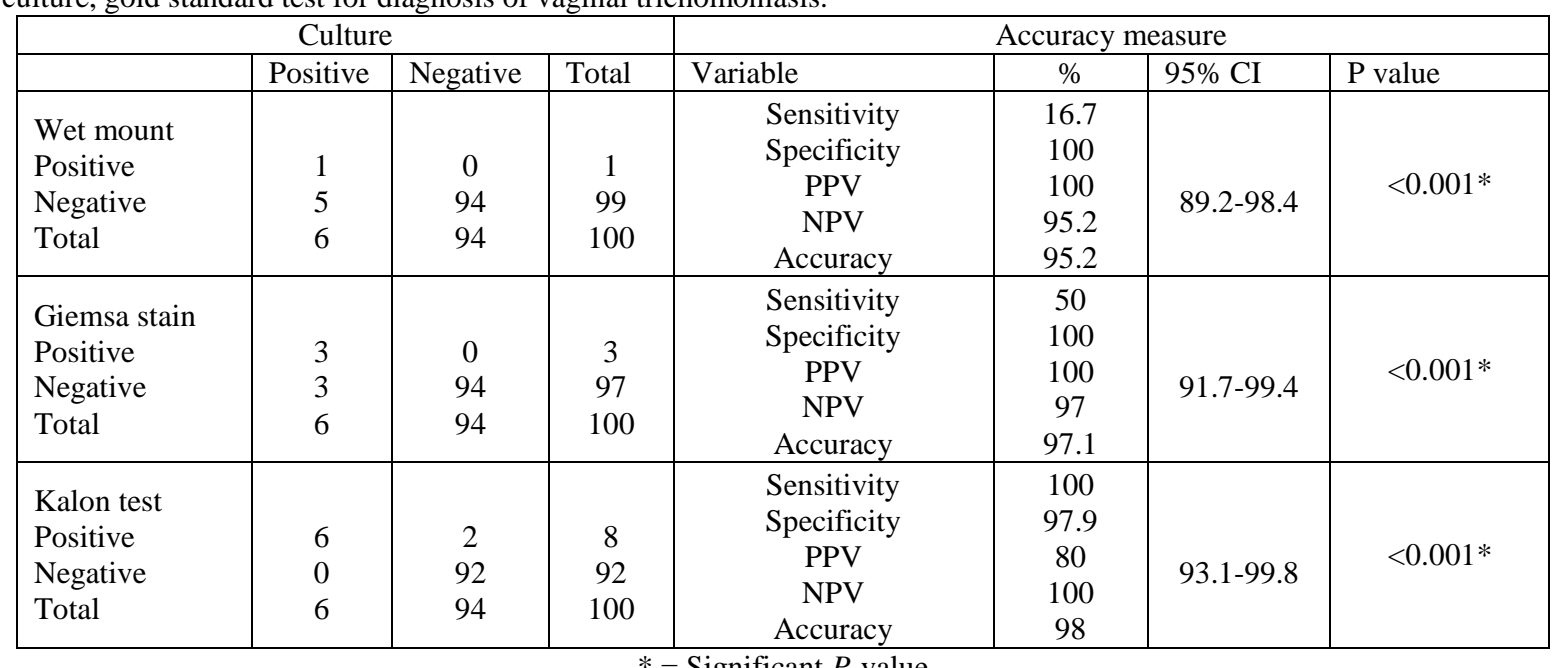

Table 2: Sociodemographic profile and clinical criteria of female patients versus Diamond's culture, gold standard test.

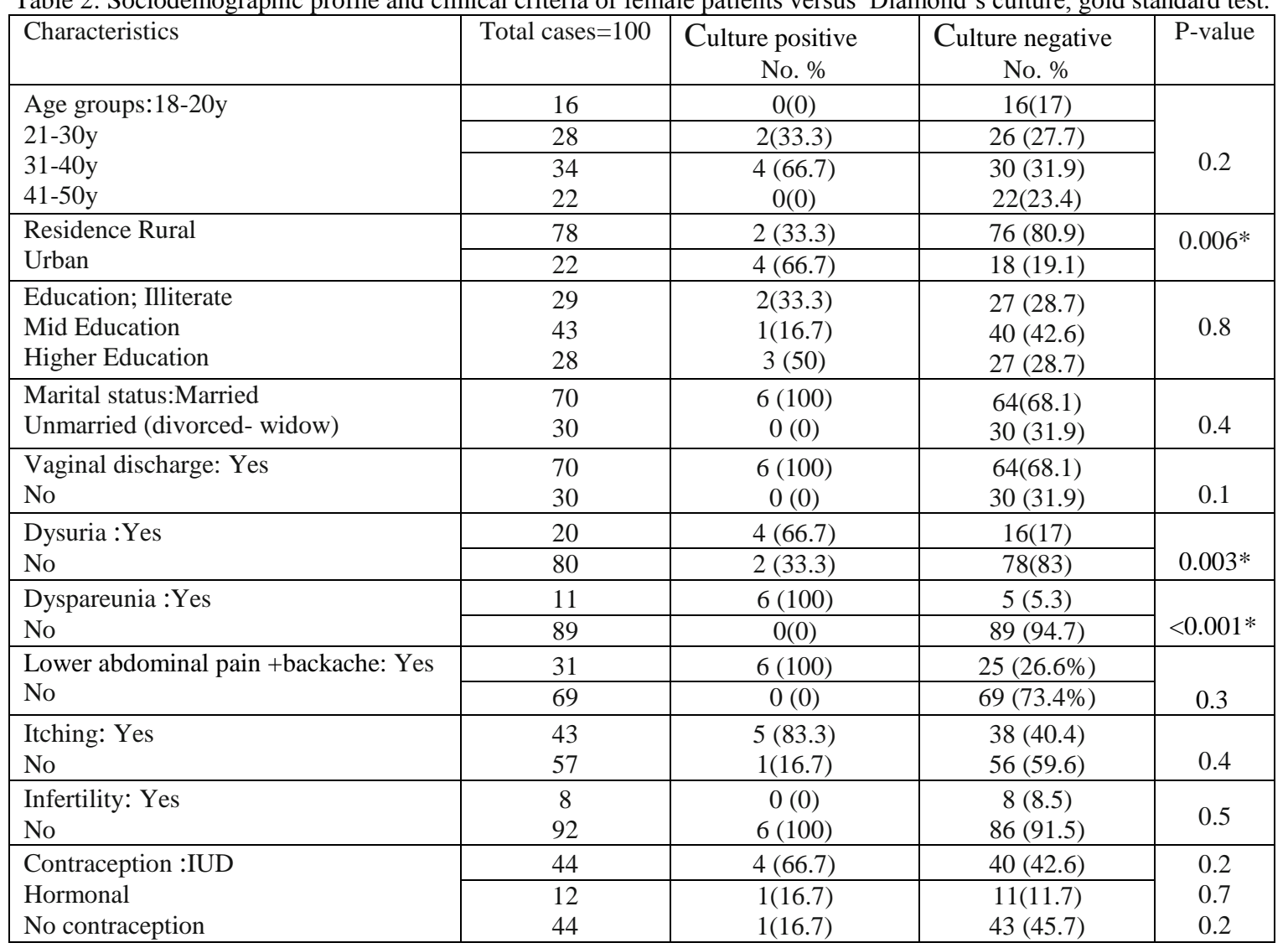

Table 3 Corrected as followed by its complete cells:

\begin{tabular}{|l|l|l|l|}
\hline Characteristics & Total no. of cases & Positive cases (\%) & P-value \\
\hline Yellowish greenish & 14 & $6(75)$ & \\
\cline { 1 - 3 } Yellowish & 26 & $2(25)$ & \multirow{3}{*}{$<0.001 *$} \\
\cline { 1 - 3 } Yellowish white/watery/bloody & 30 & $0(0)$ & \\
\hline Total & 70 & $8(100)$ & \\
\hline
\end{tabular}




\section{Discussion}

In the present study, prevalence of $T$. vaginalis infection among suspected women was $8 \%$ by all methods. This result of some reports in Egypt, which recorded that the prevalence rates among symptomatic women from Cairo, Benha and El-Minia were $10.16 \%, 11 \%$ and $12.7 \%$, respectively (Zaki et al, 2011; Hussein et al, 2015; Gabr et al, 2006). Higher prevalence rates of infection were reported from the cities of Cairo, Mansoura and Alexandria with prevalence 23\%, 36\%, 79.16\% and 91.3 (Elsherif and Youssef, 2013; Aboulghar et al, 2009; Hegazy et al, 2009; Negm and Abd El-Haleem, 2004) respectively. Lower prevalence rate of $5 \%$ was reported from Cairo and Ismailia by both Mahmoud et al. (2015) and El-Moamly and Rashad, (2008) respectively. The global prevalence estimates among suspected patients ranged from $0.9 \%$ to $80 \%$ (Valadkhani et al, 2008; Javanbakht et al, 2013).

The epidemiology of trichominiasis and disparity between different studies was changeable and may be attributed to sociocultural factors in different variable communities. Many other factors may affect accurate diagnosis and prevalence variations including variations in the selected study population, age, menstrual cycle phase, the number of sexual partners, sexual activity, other STIs, specimen type, collection and processing, available diagnostic and laboratory techniques and test interpretation (Fernando et al, 2012; Hussein et al, 2015).

In this study, $4(50 \%)$ of positive cases were patients represented by manifestations of typical vaginal trichomoniasis (vaginal discharge, itching, dyspareunia, lower abdominal pain associated with backache and dysuria). Meanwhile, in symptomatic patients with partial clinical picture, the rate of detected positive infection falls down to $25 \%$ in patients who suffering from vaginal discharge only, on the other hand another two cases $(25 \%)$ of positive cases were found inpatients complaining of both vaginal discharge and itching. There weren't any positive detected cases in patients suffering from itching alone. This result comes in accordance with results of Hussein et al. (2015) and Valadkhani et al. (2008). They had declared that combined vaginal discharge, itching and dysuria were the main complaints in $50 \%$ and $52 \%$ of $T$. vaginalis infected patients, respectively.

In this work, the highest rate of positivity (75\% of positive cases) was detected among women complaining of malodorous frothy purulent yellowish greenish vaginal discharge, however $(25 \%)$ of them were complaining of non-purulent yellowish discharge. These results are higher than but in agreement with results of Fernando et al. (2012) and Hussein et al. (2015) who detected that a characteristic frothy yellowish, and malodorous discharge in $47 \%$ and $41 \%$ of women respectively.

The results showed that except for patients living in urban areas with suggestive dysuria or dyspareunia or both there isn't any association between T.vaginalis infection and age, educational level, marital status and contraceptives use. This is partially agreed with others (Madhivanan et al, 2009; Fernando et al, 2012; Silva et al, 2013, Hussein et al, 2015). The Six out of eight positive cases were in the age group 31-40 years, the group of high sexual activity. This agreed with others (Dragsted et al, 2001; Aboulghar et al, 2009, Mahmoud et al, 2015; Hussein et al, 2015).

The culture results showed that infection was significantly common among women in urban areas $(P=0.006)$ that agreed with Ton $\mathrm{Nu}$ et al. (2015). But, Dahab et al. (2012) reported that trichomoniasis was more in rural areas than urban ones with a significance difference.

In the present study, the sociodemographic data, behavioral data and clinical manifestations were debatable. Some authors related between trichomoniasis and the race/ ethnicity (Hollman et al, 2010), age (Krashin et al, 2010), marital status (Dahab et al, 2012), education, presence of vaginal discharge, 
use of condom/IUD, (Eshete et al, 2013), and history of STIs and drug use (Miranda $e t$ $a l, 2014)$.

In the present study, $8 / 100$ suspected patients $(8 \%)$ was positive for trichomoniasis. Kalon Latex agglutination test detected all positive, but only 6 cases $(75 \%)$ were positive by Diamond's culture. Three cases and one case $(37.5 \% \& 12.5 \%)$ were positive by Giemsa staining and wet mount respectively.

The direct wet smear is a rapid, cheap, practical and simple technique but with lowest sensitivity. This might be due to trophozoite deterioration and loss of its jerky movement, retraction of its flagella making it more rounded. Thus, became difficult to detect in vaginal with epithelial cells and/or leucocytes. In this study, one case was positive by direct wet mount. This result agreed with others who showed that culture method was more sensitive than wet mount (Gavgani et al, 2008; Aboulghar et al, 2009; Zaki et al, 2011; Patil et al, 2013; Mahmoud et al, 2015; Hussein et al, 2015).

Giemsa staining is more accurate method than direct wet mount but still less sensitive than culture method. Radonjic et al. (2006) and Ojuromi et al. (2007) reported the same result. In the present study, it was noticed that Giemsa staining detected 3 cases only. This is may be attributed to the usual damage and loss of most trophozoites from the slide during processing and this is accepted by other studies (Aboulghar et al, 2009; Mahmoud et al, 2015). On the other side, Hussein et al. (2015) reported that Giemsa staining could give false positive results being its diagnosis relies only on shape and size not on motility of the trophozoite as in cases of culture and wet mount techniques.

In this study, culture is considered to be the reference test and the "gold standard" to which findings of other tests performed were compared. Radonjic et al. (2006) had confirmed that culture still remains the most sensitive and reliable method in the diagnosis of $T$. vaginalis infection. The modified Diamond culture was considered the gold standard test (Ojurom et al, 2007; Domeika et al, 2010; Hussein et al, 2015; Mahmoud et al, 2015). Meanwhile other researchers depended on diagnostic PCR as the gold standard reference test (Saleh et al, 2014). Nevertheless, Diamond culture might unavailable in most laboratories as it is expensive, and requires daily follow up for a week to detect growth, and time consuming. In many of the developing countries, patients might not visit the health facility for their culture results due to substantial cost, thus prolongation of the infection led more complications (Patel et al, 2000).

In this study, wet mount, Giemsa stain and Kalon latex agglutination test gave sensitivities of $16.7 \%, 50 \% \& 100 \%$ and the specificities of $100 \%, 100 \%$ \& $97.9 \%$ respectively. Mahmoud et al. (2015) reported that the sensitivity and specificity of latex test versus culture as standard golden test were $100 \%$ and $97.9 \%$ respectively.

The Kalon Latex test detected 8 positive cases. But, Diamond culture gave 6 positive cases. However, the confirmatory tests that might be more sensitive than culture should be performed as PCR to evaluate if the extra 2 cases detected by the Kalon test are truly false positive results or not as done previously by Saleh et al. (2014) who confirmed that latex agglutination test gave false positive results as compared with Diamond's media and PCR positive. The Kalon Latex test gave a high sensitivity when compared to culture but its positive result was weak to confirm infection $(\mathrm{PPV}=80 \%)$, meanwhile its negative result excluded the infection $(\mathrm{NPV}=100 \%)$.

\section{Conclusion}

Trichomoniasis should be included in the control programs of sexually transmitted diseases and screening tests should be done routinely to detect such infection. Sociodemographic predictors do not have a great conflict on the prevalence among different Egyptian women. Laboratory investigations are essential for accurate diagnosis. Although wet mount examination is not a sensi- 
tive technique yet, it is a good positive one, sonegative samples should be reexamined by more sensitive methods. Staining may be helpful in cases of heavy infection. Latex agglutination is a highly sensitive, simple and rapid screening test.

Competing interests: The authors declared that they neither had any competing interest nor received any fund.

\section{Acknowledgements}

The authors would like to thank the of Gynecology and Obstetrics Staff members for their cooperation and sensor efforts and the Parasitology Staff members for allowing all available resources Thanks are also due to the patients who participated in this study.

\section{References}

Aboulghar, MM, Aboushady, OM, Ahmed, JA, Hanafy, NA, 2009: Diagnosis of Trichomonas vaginalis infection in women of childbearing age at a university setting using OSOM A New Diagnostic Technique. Egypt. J. Med. Microbiol. 18, 3:51-6.

Bachmann, L, Hobbs, M, Sena, A, Sobel, J, Schwebke, J, et al, 2011: Trichomonas vaginalis genital infections: Progress and challenges. Clin. Infect. Dis. 53, 3:S160-72.

Chalamilla, G, Mbwana, J, Mhalu, F, Mmari, E, Majigo, M, et al, 2006: Patterns of sexually transmitted infections in adolescents and youth in Dares Salaam, Tanzania. BMC Infect. Dis 6:22-8.

Chesson, HW, Blandford, JM, Pinkerton, SD, 2004: Estimates of the annual number and cost of new HIV infections among women attributable to trichomoniasis in the United States. Sex. Transm. Dis. 31, 9:547-51

Cotch, MF, Joseph, P, Nugent, I, Hillier, SL, Gibbs, RS, et al, 1997: Trichomonas vaginalis associated with low birth weight and preterm delivery. Sex. Transm. Dis. 24, 6:353-60.

Dahab, MM, Koko, WS, Osman, EE, Hilali, AH, 2012: Prevalence and transmission of Trichomonas vaginalis infection among women in Khartoum State, Sudan. J. Publ. Hlth. Epidemiol. 4, 2:34-38.

Darani, HY, Ahmadi, F, Zabardast, N, Yousefi, HA, Shirzad, H, 2010: Development of a latex agglutination test as a simple and rapid method for diagnosis of Trichomonas vaginalis infection. Avicenna J. Med. Biotechnol. 2:63-66.
Domeika, M, Zhurauskaya, L, Savicheva, A, Frigo, N, Sokolovskiy, EA, Halle, A, et al, 2010: Guidelines for the laboratory diagnosis of trichomoniasis in East European countries. J Eur. Acad. Dermatol. Venereol. 24:1125-34.

Dragsted, DM, Farholt, S, Lind, I, 2001: Occurrence of trichomoniasis in women in Denmark, 1967-1997.Sex.Transm. Dis 28: 326-9.

El-Moamly, A, Rashad, S, 2008: Trichomonas vaginalis antigens in vaginal and urine specimens by immunochromatography, compared to culture and microscopy. J. Egypt. Soc. Parasitol. $38,2: 573-84$

Elsherif, RH, Youssef, MA, 2013: Real-time PCR improves detection of $T$. vaginals compared to conventional techniques. Comp. Clin. Pathol. 22, 2:295-300.

Eshete, A, Mekonnen, Z, Zeynudin, A, 2013: Trichomonas vaginalis Infection among Pregnant Women in Jimma University Specialized Hospital, Southwest Ethiopia. ISRN Infect. Dis. 2:1-5.

Fernando, SD, Herath, S, Rodrigo, C, Rajapakse, L, 2012: Clinical features and sociodemogra- phicfactors affecting $T$. vaginalis infection in women at-tending a central sexually transmitted diseases clinic in Sri Lanka. Indian J Sex Transm Dis. 33, 1:25-31.

Gabr, NS, Kamal, AM, Mohamed, RT, Abdel Wahab, SF, 2006: Sensitivity and specificity of wet mount, culture and PCR in diagnosing $T$. vaginalis infection in females attending the gynecology clinic of Minia University Hospital. Minia. Med. Bull. 17:1-8.

Gavgani, AM, Namaz, A, Ghazanchaei, A, Alizadeh, S, Sehhati, F, et al, 2008: Prevalence \& risk factors of trichomoniasis among women in Tabriz. Iran. J. Clin. Infect. Dis. 2833, 2:67-71.

Harp, DF, Chowdhury, I, 2011: Trichomoniasis: evaluation to execution. Eur. J. Obstet. Gyn. Reprod. Biol. 157:3-9.

Hegazi, M, Elbahey, M, Makhlouf, L, ElHamshary, E, Dawoud, H, et al, 2009: Polymerase chain reaction versus conventional methods in the diagnosis of vaginal trichomoniasis. J. Egypt. Soc. Parasitol. 39, 1:11-21

Hollman, D, Coupey, SM, Fox, AS, Herold, BC, 2010: Screening for Trichomonas vaginalis in high risk adolescent females with a new transcription-mediated nucleic acid amplification test (NAAT): associations with ethnicity, symptoms, and prior and current STIs. J. Pediatr. Adolesc. Gynecol. 23: 312-6. 
Huppert, J, Mortensen, J, Reed, J, Kahn, J, Mille,r W, Hobbs, M, 2007: Comparison of diagnostic methods for Trichomonas vaginalis. J. Adoles. Hlth. 40, 2:S8-12.

Hussein, AH, Saleh, MH, Nagaty, IM, Ghieth, KA, El-Azab, NA, 2015: Prevalence, Clinical Criteria and Sociodemographic Predictors of Trichomonas vaginalis infection in suspected Egyptian women, using direct diagnostic techniques. Iran J. Parasitol. 10, 3:432-40

Javanbakht, M, Stirland, A, Stahlman, Sh, Smith, LV, Chien, M, et al, 2013: Prevalence and factors associated with Trichomonas vaginalis infection among high-risk women in Los Angeles. Sex. Transm. Dis 40, 10:804-7.

Kaul, P, Gupta, I, Sehgal, R, Malla, N, 2004: Trichomonas vaginalis: random amplified polymer-phic DNA analysis of isolates from symptomatic and asymptomatic women in India. Parasitol. Int. 262:253-5.

Krashin, JW, Koumans, EH, Bradshaw, AC, Braxton, JR, Secor, WE, et al, 2010: $T$. vaginalis prevalence, incidence, risk factors and antibiotic-resistance in 306 adolescent population. Sex. Transm. Dis. 37:440-4.

Mabey, D, Ackers, J, Adu-Sarkodie, Y, 2006: Trichomonas vaginalis infection. Sex. Transm. Infect. 82, 4:S26-7.

Madhivanan, P, Bartman, M, Pasutti, L, Krupp, K, Arun, A, et al, 2009: Prevalence of Trichomonas vaginalis infection among young reproductive age women in India: implications for treatment and prevention. Sex Hlth. 6, 4:339-44. Mahmoud, A, Sherif, NA, Abdella, R, ElGenedy, AR, El Kateb, AY, Askalan,i ANH, 2015: Prevalence of Trichomonas vaginalis infection among Egyptian women using culture and Latex agglutination: cross-sectional study. BMC Women's Health 15:7, 1-6

McClelland, RS, Sangaré, L, Hassan, WM, Lavreys, L, Mandaliya, K, et al, 2007: Infection with Trichomonas vaginalis increases the risk of HIV-1 acquisition. J. Infect. Dis. 195, 5:698-702

Miranda, AE, Pinto, VM, Gaydos, CA, 2014: Trichomonas vaginalis infection among young pregnant women in Brazil. BJID 18, 6:669-7.

Moodley, P, Wilkinson, D, Connolly, C, Moodley, J, Sturm, AW, 2002: Trichomonas vaginalis is associated with pelvic inflammatory disease in women infected with human immunodeficiency virus. Clin. Infect. Dis. 34:519-22.

Negm, AY, Abd El-Haleem, DA 2004: Detec- tion of trichomoniasis in vaginal specimens by both conventional and modern molecular tools. J. Egypt. Soc. Parasitol. 34, 2:589-600.

Pararas, M, Skevaki, C, Kafetzis, D, 2006: Preterm birth due to maternal infection: causative pathogens and modes of prevention. Eur. J. Clin. Microbiol. Infect. Dis. 25, 9:562-9

Patel, SR, Weise, W, Patel, SC, Ohl, C, Byrd, JC, et al, 2000: System review of diagnostic tests for T. vaginalis. Infect. Dis. Obstet. Gynecol. 8:248-57.

Patil, MJ, Nagamoti, JM, Metqud, SC, 2012: Diagnosis of $T$. vaginalis from vaginal specimens by wet mount microscopy, In: Pouch TV culture system and PCR. J. Glob. Infect. Dis. 4, 1:22-5.

Ojuromi, OT, Oyibo, WA, Tayo, AO, Ibidapo, AI, Fagbenro-Beyioku, AF, et al, 2007: Reliance on microscopy in T. vaginalis diagnosis and its prevalence in females presenting with vaginal discharge in Lagos, Nigeria. J. Infect. Dev. Ctries 1, 2:210-3.

Radonjic, IV, Dzamic, AM, Mitrovic, SM, Arsenijevic, A, Popadic, DM, et al, 2006: Diagnosis of T. vaginalis infection: The sensitivities and specificities of microscopy, culture and PCR assay. Eur. J. Obst. Gyn. Reprod. Biol. 126, 1:116-20.

Saleh, AM, Abdalla, HS, Satti1, AB, Babiker, SM, Gasim, GI, et al, 2014: Diagnosis of Trichomonous vaginalis by microscopy, latex agglutination, diamond's media, and PCR in symptomatic women, Khartoum, Sudan. Diagn. Pathol. 9, 49:1-5.

Secor, WE, Meites, E, Starr, MC, Workowski, KA, 2014: Neglected Parasitic Infections in the United States: Trichomoniasis. Am. J. Trop. Med. Hyg. 90, 5:800-4.

Sherrard, J, Donders, G, White, D, Jensen, JS, 2011: European (IUSTI/WHO) guideline on the management of vaginal discharge. Int. J. STD AIDS 22:421-9.

Sibau, L, Bebb, D, Proctor, EM, Bowie, WR, 1987: Enzyme-linked immunosorbent assay for the diagnosis of trichomoniasis in women. Sex Transm Dis 14:216-20.

Silva, L, Miranda, A, Batalha, R, Montea, R, Talhari, S, 2013: Trichomonas vaginalis and associa- ted factors among women living with HIV/AIDS in Amazonas, Brazil. Braz. J. Infect. Dis. 17, 6:701-3.

Stark, JR, Alderete, JF, Judson, G, Mundodi, V, Kucknoor, AS, et al, 2009: Prospective stu- 
dy of Trichomonas vaginalis infection and prostate cancer incidence and mortality: Physicians' health study. J. Natl. Canc. Inst. 101, 20:1406-11 Sutcliffe, S, Giovannucci, E, Alderete, JF, Chang, TH, Gaydos, CA, et al, 2006: Plasma antibodies against Trichomonas vaginalis and subsequent risk of prostate cancer. Canc. Epidemiol. Biomarkers Prevent. 15, 5:939-45

Ton Nu, PA, Nguyen, VQH, Cao, NT, Dessì, D, Rappelli, P, et al, 2015: Prevalence of Trichomonas vaginalis infection in symptomatic and asymptomatic women in Central Vietnam. J. Infect. Dev. Ctries. 9, 6:655-60

Twu, O, Dessí D, Vu, A, Mercer, F, Stevens, GC, De Miguel, N, Fiori, PL, 2014: Trichomonas vaginalis homolog of macrophage migration inhibitory factor induces prostate cell growth, 401invasiveness, and inflammatory responses. Proc. Natl. Acad. Sci. 111, 22:8179-84

Valadkhani, Z, Assmar, MB, Esfandiari, B, Amirkhani, A, Hassan, N, et al, 2008: Trichomoniasis in asymptomatic patients. Iran J. Publ. Hlth. 37, 3:113-7

Wang, CC, McClelland, R, Reilly, M, Overbaugh, J, et al, 2001: Effect of treatment of vaginal infections on shedding of human immunodeficiency virus type 1. J. Infect. Dis. 183, 7:101722

WHO, 2012: Global incidence and prevalence of selected curable sexually transmitted infections: Available athttp://www.whoint/ reproductivehealth/publications/rtis/stisestimates/ en.

Zaki, MM, Moussa, H, Hassanin, OM, 2011: Evaluation of the OSOM Trichomonas rapid test for detection of trichomoniasis. PUJ 4, 2:177-84.

\section{Explanation of figures}

Fig. 1: Comparison of Receiver operating characteristic curve (ROC) for diagnostic performance of wet mount, Giemsa staining technique and Kalon latex agglutination test as referred to in-vitro culture in diagnosis of vaginal trichomoniasis.

Fig. 2: Trichomonas vaginalis "pear" shaped flagellated trophozoite after staining with Giemsa stain by $\times 100$.

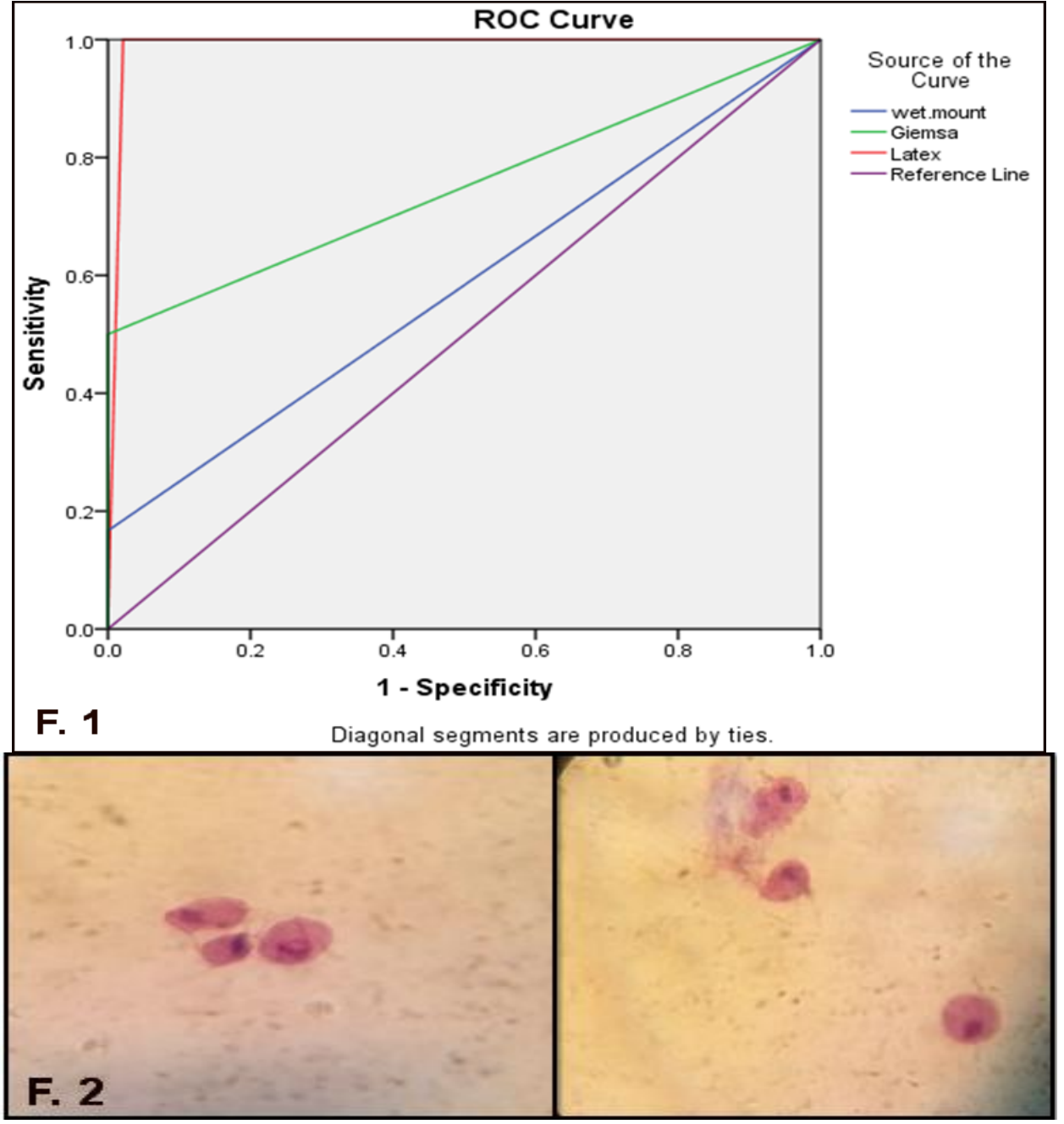

